\title{
Ökonomische Theorie des Rechts, beschränkte Rationalität und französischer Kündigungsschutz ${ }^{1}$
}

\section{Einführung}

\subsection{Unsicherheit, Risiko und Regulierung im Arbeitsrecht}

Eines der Risiken von Beschäftigungsverhältnissen besteht darin, daß durch Vorschriften des Arbeitsrechts Arbeitskosten zu Fixkosten werden oder daß Sonderbelastungen für die Beendigung des Arbeitsverhältnisses anfallen. Sieht das Arbeitsrecht Beschränkungen in bezug auf die Vereinbarung von Befristungen in Arbeitsverträgen vor oder reguliert es Kündigungsgründe und/oder -fristen, stellt sich der Abschluß eines Arbeitsvertrages aus Sicht des Arbeitgebers als ein risikoreiches Unterfangen dar. Es geht um das Problem, daß die Möglichkeit der Beendigung von Arbeitsverhältnissen durch rechtliche Regelungen eingeschränkt oder verteuert wird. Enthalten diese Regelungen dann Generalklauseln oder breite, auslegungsbedürftige Rechtsbegriffe, ist deren Interpretation durch die Gerichte schwer abschätzbar. Aus Sicht von Arbeitgebern bedeutet dies, daß die Fertigungskapazität gegebenenfalls nur mit erheblichen zeitlichen Verzögerungen an den Bedarf angepaßt werden kann.

In einem solchen Regulierungsumfeld ist davon auszugehen, daß Arbeitgeber das existierende arbeitsrechtliche Instrumentarium - insbesondere die Kündigungsregelungen - zielgerichtet zur Wahrung ihrer Interessen einsetzen werden. Dies wiederum setzt voraus, daß sie Wirkungen von arbeitsgerichtlichen Streitverfahren im Bereich des Kündigungsschutzes richtig einschätzen können. Lassen sich hier signifikante Abweichungen des tatsächlichen Verhaltens von einem als ideal angenommenen Rationalverhalten feststellen, gibt dies Anlaß, das Instrumentarium des verhaltenswissenschaftlich orientierten (behavioristischen) Ansatzes zur ökonomischen Untersuchung rechtlicher Problemstellungen (behavioral law and economics) ${ }^{2}$ einzusetzen, um solche Abweichungen zu erklären und damit möglicherweise auch zu überlegenen Problemlösungen beizutragen.

\subsection{Besonderheiten des Arbeitsrechts}

Das Arbeitsrecht zeichnet sich gegenüber vielen anderen Rechtsgebieten dadurch aus, daß der Anteil des Richterrechts im Verhältnis zum legislativ gesetzten Recht höher

1 Überarbeiteter Diskussionsbeitrag zu »Litigations in Labor Law: Intuitive vs. Reflective Judgements« von Bruno Deffains und Samuel Ferey Workshop »Uncertainty, Risk and Regulation: The Behavioral Law and Economics Perspective«, 16./17. Juni 2006, Technische Universität Berlin / Humboldt-Universität zu Berlin.

2 Vgl. u.a. Sunstein, Cass R. (Hrsg.), Behavioral Law \& Economics, Cambridge, England, 2000; Jolls, Christine/Sunstein, Cass R./Thaler, Richard H., A Behavioral Approach to Law and Economics, in: Sunstein, Cass R. (Hrsg.), Behavioral Law \& Economics, Cambridge, England, 2000, S. 13 - 58; aus institutionenökonomischer Sicht: Hodgson, Geoffrey M., Economics and institutions, A manifesto for a modern institutional economics, Oxford u.a., 1988, S. $106 \mathrm{ff}$. 
ist. ${ }^{3}$ Das ist in Deutschland, in Frankreich und im europäischen Gemeinschaftsrecht zu beobachten. Der Grund dafür mag darin liegen, daß es aus Sicht politischer Entscheidungsträger vorteilhaft erscheint, politisch kontroverse Entscheidungen solchen Personen zu überlassen, die nicht der Abwahldrohung im demokratischen System ausgesetzt sind, also den Richtern. Also neigen Legislativorgane im Arbeitsrecht oft dazu, breite Ermessensspielräume vorzusehen und damit faktisch Rechtsetzungsbefugnis an die Gerichte zu delegieren. Das eröffnet nun den in der Judikative tätigen Akteuren die Möglichkeit, die Interpretation arbeitsrechtlicher Vorschriften für normative Zielsetzungen gezielt einzusetzen. Eine der möglichen Zielsetzungen ist die Verteidigung von Arbeitnehmern gegen die als übermächtig empfundenen Interessen von Arbeitgebern. ${ }^{4}$

\subsection{Probleme beschränkter Rationalität in arbeitsgerichtlichen Streitverfahren}

Wird in Gerichtsentscheidungen, in denen der Arbeitnehmer als die >schwächere < Vertragsseite eines Arbeitsvertrags geschützt wird, übersehen, daß solche Entscheidungen zwar für die direkt Betroffenen Vorteile bringen mögen, für die Arbeitnehmer aber insgesamt mit erheblichen Nachteilen verbunden sein können (etwa weil die strukturelle Arbeitslosigkeit erhöht wird), kann es sich um Vorurteile oder Fehleinschätzungen (biases) handeln. Auch die Vertragsparteien von Arbeitsprozessen können Fehleinschätzungen unterliegen, wenn sie etwa Kündigungen aus persönlichem Grund einer Kündigung aus wirtschaftlichen Erwägungen systematisch deshalb vorziehen, weil sie annehmen, daß Gerichte letztere Fälle strenger behandeln. Stellt sich dann aber heraus, daß Arbeitgeber das Risiko von Kündigungsklagen mit persönlichem Kündigungsgrund unterschätzen, mag sich die anscheinend rationale Strategie im nachhinein als falsch herausstellen. Solche Fehleinschätzungen können ihre Ursache in einer verzerrten Problemerfassung durch die Parteien haben und damit in deren beschränkter Rationalität. Mit solchen Verzerrungen und daraus resultierenden Fehleinschätzungen befaßt sich - wie oben ausgeführt - die behavioristische ökonomische Analyse des Rechts (behavioral law and economics).

Der Beitrag von Deffains und Ferey steht auf dem Boden dieses Ansatzes. Es geht hier um ein Spezialproblem des beschränkt rationalen Verhaltens der an einer arbeitsrechtlichen Streitigkeit beteiligten Akteure. Reaktionen der verschiedenen Akteure auf Regelungen des Kündigungsschutzrechts können als Beispiel für eine solche Abweichung der Akteure vom Modell des Rationalverhaltens untersucht werden. Das französische Kündigungsschutzrecht eignet sich für eine solche Untersuchung deshalb, weil mit dem sehr unbestimmten Ausdruck >cause réelle et sérieuse< in Art. L 122-14-3 des Code du Travail den Gerichten erhebliche Ermessensspielräume einge-

3 Zur Rolle von Gerichten als »Ersatzgesetzgeber « im Arbeitsrecht: Rüthers, Bernd/Birk, Axel, Rechtstheorie, 2. Aufl., München, 2005, S. 178 - 180, insbes. S. 179; für das europäische Gemeinschaftsrecht: Rebhahn, Robert, Europäisches Arbeitsrecht, in: Riesenhuber, Karl (Hrsg.), Europäische Methodenlehre, Grundfragen der Methoden des Europäischen Privatrechts, Berlin, 2006, S.330 - 356, bes. S. 347 f.

4 Vgl. zum Argument favor laboris: Rebhahn, a.a.O. (Fn. 2), S. 341 - 344. 

schutz

räumt werden. ${ }^{5}$ Es geht dann um zwei Fragen, nämlich erstens, ob Richter beschränkt rational handeln, und zweitens, ob Arbeitgeber in Bezug auf Kündigungen falsche Entscheidungen aufgrund beschränkt rationalen Verhaltens treffen.

\subsection{Inhalt und Aufbau des Diskussionsbeitrags}

Deffains und Ferey unternehmen in ihrer Untersuchung von Paradoxien in Arbeitsgerichtsstreitigkeiten den Versuch, mit Hilfe des Instrumentariums der behavioristischen ökonomischen Analyse des Rechts Erklärungen für das Verhalten der streitenden Parteien zu geben, die jenseits dessen liegen, was herkömmliche ökonomische Analysen zu sagen haben, die von der Annahme des Rationalverhaltens der Parteien ausgehen.

Es stellt sich aber die Frage, ob es zur Erklärung der von den Autoren untersuchten Paradoxien einer Modifizierung der Annahme des Rationalverhaltens im Sinn behavioristischer Ansätze bedarf. Dahinter steht ein grundsätzliches Problem, wie sich aus Sicht der ökonomischen Theorie Probleme der Interpretation rechtlicher Vorschriften mit breiten Ermessensspielräumen - wie etwa im Arbeitsrecht - darstellen. ${ }^{6}$ Es ist zu klären, ob zwischen der rechtswissenschaftlichen und der ökonomischen Herangehensweise grundsätzlich eine Unvereinbarkeit besteht oder ob sich zwischen beiden eine Brücke schlagen läßt. Hier soll der weiteren Frage nachgegangen werden, ob sich an der Fallkonstellation etwas ändert, wenn Gerichte sich vermehrt Interpretationsmethoden zuwenden, die ökonomische Ansätze bei der Auslegung rechtlicher Vorschriften ins Spiel bringen.

\section{Paradoxien im Verhalten französischer Arbeitgeber in arbeitsrechtlichen Kündi- gungsverfahren}

Nach dem französischen Arbeitsgesetz (Code du Travail) wird zwischen Kündigungen durch den Arbeitnehmer (démission) und solchen durch den Arbeitgeber (licenciement) unterschieden. Die Kündigung durch Arbeitgeber muß sich nach Art. L 122-143 Code du Travail auf einen »ernsthaften « Grund (cause réelle et sérieuse) stützen. ${ }^{7}$ Dieser kann in der Person des Arbeitnehmers liegen (motif personnel) oder es können wirtschaftliche Gründe geltend gemacht werden. Unter einer Kündigung aus wirtschaftlichen Gründen (licenciement pour cause économique) ist nach Art. 321-1 in der Fassung des Gesetzes vom 2.8.1989 eine Entlassung zu verstehen, die nicht in der Person des Arbeitnehmens begründet ist, sondern aus dem Wegfall oder der Umwandlung des Arbeitsvertrags resultiert, insbesondere infolge wirtschaftlicher Schwierigkeiten oder technischen Fortschritts. ${ }^{8}$ Im Gesetz werden aber keine Kriterien genannt, die die

5 Hübner, Ulrich / Constantinesco, Vlad, Einführung in das französische Recht, 4. Aufl., München 2001, S. 231; Sonnenberger; Hans-Jürgen/Autexier, Christian, Einführung des französische Recht, 3. Aufl., Heidelberg, S. 222.

6 Vgl. Rüthers/Birk. a.a.O. (Fn. 2), S. 141.

7 Nachweise in Fn. 4.

8 Vgl. Sonnenberger, Hans Jürgen, Französisches Handels- und Wirtschaftsrecht, 2. Aufl., Heidelberg, 1991, S. 278, Rndr. V 30. 
>nature réelle et sérieuse< der wirtschaftlichen Gründe näher bestimmen. ${ }^{9}$ Damit ist es eine Aufgabe der Rechtsprechung, den Begriff zu konkretisieren.

In ihrem Referat machen Deffains und Ferey darauf aufmerksam, daß die französischen Gerichte in der Zulassung von Kündigungen aus wirtschaftlichen Gründen sehr zurückhaltend sind. Dies mag damit zusammenhängen, daß die Gerichte mit einer solchen Rechtsprechung im konkreten Fall bestehende Arbeitsverhältnisse sichern und gegebenenfalls dann davon ausgehen, daß sie damit einen Beitrag zur Senkung der Arbeitslosigkeit bzw. einen Beitrag zur Verhinderung der Erhöhung der Arbeitslosigkeit leisten. Aus Sicht von Arbeitgebern, die ein Interesse daran haben, die Zahl der von ihnen angebotenen Arbeitsplätze an sich ändernde wirtschaftliche Verhältnisse anzupassen, bedeutet eine solche Rechtsprechung eine Kostenbelastung, da variable Kosten in fixe Kosten umgewandelt werden.

Es besteht - Rationalverhalten unterstellt - ein Anreiz, nach anderen Kündigungsmöglichkeiten Ausschau zu halten. Es kommt möglicherweise in konkreten Fällen in Betracht, statt einer Kündigung aus wirtschaftlichen Gründen eine solche aus persönlichen Gründen auszusprechen. Auch hier geht es um einen ernsthaften Grund (cause réelle et serieuse), der in jedem einzelnen Fall vorliegen muß. Substituieren nunmehr Arbeitgeber Kündigungen aus wirtschaftlichen Gründen durch solche aus persönlichen Gründen, könnten sie damit möglicherweise in einer größeren Zahl von Fällen erfolgreich sein und somit Kosten sparen. Es ist aber auch möglich, daß sie das Risiko von Kündigungen, die auf persönliche Gründe abstellen, systematisch zu tief einschätzen und dann letztlich mit ihrer Substitutionsstrategie sogar höhere Kosten in Kauf zu nehmen haben. Dies wäre dann eine Fallkonstellation, die sich nur erklären ließe, wenn man die Rationalitätsannahme verändert und mit der behavioristischen ökonomischen Analyse des Rechts davon ausgeht, daß es bei bestimmten Konstellationen von einem Überoptimismus (optimistic bias) ${ }^{10}$ der Akteure auszugehen ist.

Geht man von der Annahme des Rationalverhaltens aus, so sind sehr wohl Substitutionsprozesse zwischen Kündigungen aus wirtschaftlichen und solchen aus persönlichen Gründen denkbar, bei denen im Ergebnis bei Kündigungen aus persönlichen Gründen die prozentuale Häufigkeit von gerichtlichen Streitigkeiten höher liegt als bei Kündigungen aus wirtschaftlichen Gründen. Dies ist deshalb so, weil durch den Substitutionsprozeß sich die betreffenden Häufigkeiten verschieben. Wird im Ausgangsfall angenommen, daß die betreffende Häufigkeit von gerichtlichen Streitigkeiten bei Kündigungen aus wirtschaftlichen Gründen bei $50 \%$, bei solchen aus persönlichen Gründen bei $20 \%$ liegt, und ist nach dem Substitutionsprozeß die Häufigkeit bei Kündigungen aus persönlichen Gründen auf 30\% gestiegen, die bei Kündigungen aus wirtschaftlichen Gründen aber auf 20\% gesunken, so liegt die Vermutung nahe, daß sich bei Kündigungen aus persönlichen Gründen nun auch solche Fälle befinden, in denen es eigentlich um wirtschaftliche Gründe geht, persönliche Gründe aber nachgeschoben werden. Für die von solchen Kündigungen Betroffenen ist es dann rational, die betreffende Kündigung gerichtlich

9 Damit handelt es sich mit Rüthers/Birk um eine »kalkulierte Unbestimmtheit und Offenheit von Gesetzesbegriffen, a.a.0, (Fn. 2), S. 141.

10 Vgl. Sunstein, Cass R., Introduction, in: Sunstein, Cass (Hrsg.), a.a.O. (Fn. 1), S. 4. 

schutz

überprüfen zu lassen, zumal nunmehr bei der Auslegung der Ernsthaftigkeit der Gründe persönliche Gründe von Seiten des Gekündigten ins Spiel gebracht werden können.

Dies wird an einem hypothetischen Fall deutlich: Man nehme an, es hat vor der Substitution 500000 Fälle von Kündungen aus wirtschaftlichen Gründen und 100000 aus persönlichen Gründen gegeben. Legt man hier die oben gemachten Annahmen bezüglich der Häufigkeit gerichtlicher Streitigkeiten zugrunde, so würde das zur Folge haben, daß 250000 Kündigungen aus wirtschaftlichen Gründen und 20000 Kündigungen aus persönlichen Gründen zu Gerichtsverfahren führen würden. Werden nun 400000 Kündigungen aus wirtschaftlichen Gründen in die Kategorie der Kündigung aus persönlichen Gründen umgeschichtet und verändern sich damit auch die Häufigkeiten der gerichtlichen Verfahren, so werden nunmehr 20000 Kündigungen aus wirtschaftlichen Gründen zu Gerichtsverfahren führen und 150000 Kündigungen aus persönlichen Gründen. Die Gesamtzahl der Kündigungen, die zu Gerichtsverfahren führen, würde damit von 270000 auf 170000 abnehmen und das, obwohl jetzt mehr Kündigungen aus persönlichen Gründen ausgesprochen werden und die Häufigkeit von Gerichtsverfahren bei diesen höher liegt als bei Kündigungen aus wirtschaftlichen Gründen. Diese Überlegungen besagen nun nicht, daß bei den Akteuren nicht Überoptimismus als eine Variante beschränkt rationalen Verhaltens im Spiel sein kann. Auch ist es denkbar, daß es sehr wohl Fallkonstellationen geben kann, die sich nur unter Zugrundelegung einer solchen Verhaltensannahme erklären lassen. Um dies festzustellen, wäre es aber notwendig, daß die Häufigkeiten vor und nach solchen Substitutionsprozessen bekannt wären. Hier aber liegt das Problem. Die Wahrscheinlichkeit vor dem Substitutionsprozeß ist nicht erfaßbar, weil hierüber nur Mutmaßungen existieren. Es sind die subjektiven Annahmen der Akteure über diese Wahrscheinlichkeiten, die einen Faktor für die Auslösung des Substitutionsprozesses darstellen.

\section{Brücken zwischen rechtswissenschafticher Interpretation unbestimmter Rechtsbe- griffe und ökonomischer Theorie}

\subsection{Das rechtswissenschaftliche Methodenproblem aus ökonomischer Sicht}

Die Ursache für das beobachtete Ausweichverhalten von Arbeitgebern, die sich aus Kostengründen für eine Auswechslung der Kündigungsgründe entscheiden, liegt in der systematischen Unsicherheit, den Ausgang von Kündigungen abzuschätzen, die sich auf wirtschaftliche Gründe (licenciement pour cause économique) stützen und bei denen ein ernsthafter Grund (cause réelle et sérieuse) vorliegen muß. Was ein solcher ernsthafter Grund ist, wird vom Gesetzgeber nicht näher bestimmt. Die Ausfüllung des Begriffs wird den Gerichten überlassen. Es geht also um das Problem, Prognosen darüber zu machen, wie Gerichte mit Ermessensspielräumen umgehen. ${ }^{11}$

11 Vgl. dazu Kirchner, Christian, Probleme von Ermessensspielräumen in der fair valueBewertung nach Internationalen Rechnungslegungsstandards, in: Zeitschrift für betriebswirtschaftliche Forschung, 2006 (Sonderheft 55/06, S. 61-78), insbes. Abschnitt 2.3 (Relevanz von Interpretationsmethoden und Meta-Regeln für die institutionenökonomische Analyse). 
Enthalten rechtliche Regelungen Ermessensspielräume, bedeutet dies aus Sicht der Regelungsadressaten, dass sie Prognosen zu erstellen haben, wie diese Regelungen auf konkrete Sachverhalte angewandt werden. Bei solchen Prognosen handelt es sich um die Ergebnisse von Wirkungsanalysen von Institutionenänderungen im Sinne der Institutionenökonomik. ${ }^{12}$ Entscheidend ist dann, wer diese Regelungen auslegt und welche Methoden der Interpretation der Regelungen eingesetzt werden. In der herkömmlichen ökonomischen Theorie des Rechts wird dies bisher wenig beachtet. ${ }^{13}$ Es wird unterstellt, daß für die Interpretation der rechtlichen Regelungen die herkömmlichen juristischen Interpretationsmethoden zur Anwendung gelangen, ohne zu fragen, welche Implikationen damit verbunden sind. Folglich ist der institutionenökonomische Ansatz hier weiter zu entwickeln.

Institutionen können als allgemeine Regelungen begriffen werden, die mit Hilfe eines Sanktionsinstrumentariums durchgesetzt werden. ${ }^{14}$ Im institutionenökonomischen Ansatz wird zuerst einmal untersucht, wie alternative Ausgestaltungen von rechtlichen Regelungen zu unterschiedlichen Wirkungen bei den Adressaten dieser Regelungen führen. Solche Wirkungsanalysen setzen nun aber voraus, dass mit Annahmen zur verwendeten Methodik bei der Interpretation dieser Regelungen gearbeitet wird.

Aus institutionenökonomischer Sicht geht es erst einmal nicht um die Qualität der verwendeten Interpretationsmethoden, sondern um eine Prognose, zu welchen Ergebnissen die tatsächlich angewandten Methoden führen werden. Die davon zu trennende Frage, die hier nicht behandelt wird, betrifft eine mögliche Verbesserung der Interpretationsmethoden durch Einfügung ökonomischer Elemente in die juristische Auslegung. ${ }^{15}$

\subsection{Herkömmliche rechtswissenschaftliche Interpretationsmethoden}

In der herkömmlichen Methodendiskussion der Rechtswissenschaft steht der Zusammenhang zwischen Normziel und gewählter Interpretation im Zentrum der teleologischen Interpretationsmethode. ${ }^{16}$ Nach der teleologischen Interpretationsmethode wird die Auslegung gesucht, die am besten dem Normzweck entspricht. Entscheidend ist dann zuerst, wie der Normzweck zu bestimmen ist. Im Kündigungsschutzrecht könnte dies etwa der Grundsatz favor laboris sein. ${ }^{17}$

12 Vgl. für viele: Richter, Rudolf/Furubotn, Eirik, Neue Institutionenökonomik, 2. Aufl., Tübingen, 2003; Voigt, Stefan, Institutionenökonomik, München 2002.

13 Vgl. etwa Kirchner, Christian, Ökonomische Theorie des Rechts, Berlin 1997.

14 Vgl. zu Durchsetzungsproblematik Weber, Max, Wirtschaft und Gesellschaft. Grundriß der verstehenden Soziologie, Tübingen, 5. Aufl. 1980, S. 17; auch Richter/Furubotn, a.a.O. (Fn. 11), S. 7.

15 Zur Entwicklung einer interdisziplinären Methodik der Interpretation rechtlicher Regelungen: Kirchner, Christian, Die ökonomische Theorie (§ 4), in: Riesenhuber, Karl (Hrsg.), Europäische Methodenlehre. Grundfragen der Methoden des Europäischen Privatrechts, Berlin, 2006, S. 23 - 48.

16 Vgl. für viele: Röhl, Klaus F., Allgemeine Rechtslehre, 2. Aufl. (2001), S. 600 - 603; Bydlinski, Franz, Juristische Methodenlehre und Rechtsbegriff, 2. Aufl. (1982), S. 435 - 463; Koch, Hans-Joachim/Rüßmann, Helmut, Juristische Begründungslehre (1982), S. 222 227; Rüthers/Birk, Rechtstheorie, 2. Aufl. (2005), S. 461 - 466.

17 Vgl. oben Abschnitt 1.2 sowie den Nachweis in Fn. 3. 

schutz

Wird dann vom Normzweck auf die angemessene Interpretation geschlossen, so kommt das Zweck-Mittel-Paradigma ins Spiel. ${ }^{18}$ Es wird angenommen, daß die Interpretation ein relevanter Faktor für die Erreichung des Normzwecks sei. Unterschiedliche Interpretationsvarianten stellen Mittel dar, die im Rahmen des Zweck-Mittel-Verhältnisses unter dem Gesichtspunkt einer möglichst genauen Zielerreichung eingesetzt werden. Dann benötigt man aber Informationen darüber, wie unterschiedliche Interpretationsvarianten sich tatsächlich auf die Zielerreichung auswirken. Es sind also in Bezug auf diese Varianten Wirkungsanalysen durchzuführen. Die Problematik der teleologischen Interpretationsmethode liegt dann zum einen darin, daß für diese Wirkungsanalysen ein entsprechender methodischer Ansatz zu entwickeln ist, der es ermöglicht, synthetisch-nomologische, also empirisch gehaltvolle, nachprüfbare Aussagen darüber zu machen, wie sich unterschiedliche Interpretationsvarianten für die Zielerreichung eignen. Die Qualität dieser - positiven - Aussagen ist zentrales Element bei der Verwendung einer teleologischen Methode der Norminterpretation. Sie wiederum hängt vom verwendeten methodischen Ansatz ab. In der Rechtswissenschaft sind Versuche unternommen worden, unter dem Stichwort $»$ Rechtsfolgenanalyse « eine Methodik für solche Wirkungsanalysen zu erarbeiten. ${ }^{19}$ Es ist jedoch nicht gelungen, einen eigenen rechtswissenschaftlichen Methodenbeitrag zu entwickeln. Vielmehr ist in dieser Diskussion vorgeschlagen worden, statt einen eigenen rechtswissenschaftlichen Ansatz zu entwickeln, auf das methodische Instrumentarium der Neuen Institutionenökonomik zurückzugreifen. ${ }^{20}$

Das Zweck-Mittel-Paradigma ist ein Optimierungsprogramm. Es fragt alternativ, welches bestmögliche Ziel mit einem gegebenen Mitteileinsatz erreicht werden kann oder wie ein gegebenes Ziel mit einem minimalen Mitteleinsatz erreicht werden kann. Damit ist es Ausdruck der ökonomischen Zweckrationalität im Sinne von Max Weber. ${ }^{21}$ Es können gegen das Zweck-Mittel-Paradigma drei grundsätzliche Einwände formuliert werden: ${ }^{22}$ (1) Die Konzentration auf die Zweck-Mittel-Problematik klammert die Zieldiskussion weitgehend aus; es wird axiomatisch argumentiert. (2) >Nebenwirkungen' der eingesetzten Mittel werden entweder nicht in Rechnung gestellt oder allenfalls als Störfaktoren gesehen. Es geht dabei um die nicht intendierten Handlungsfolgen intentionalen Tuns im Hayekschen $S_{i n n e}{ }^{23}$, die ein erhebliches Problem

18 Zum Zweck-Mittel-Paradigma: Streit, Manfred E., Theorie der Wirtschaftspolitik, 6. Aufl. (2005), S. 270; Mertens, Hans-Joachim/Kirchner, Christian/Schanze, Erich, Wirtschaftsrecht, Opladen, 2. Aufl. (1978), S. 46 f.

19 Wälde, Thomas W., Juristische Folgenorientierung, Königstein 1979; Deckert, Martina Renate, Folgenorientierung in der Rechtsanwendung, München 1995.

20 Vgl. Kirchner, Christian, Folgenberücksichtigung bei judikativer Rechtsfortbildung und Ökonomische Theorie des Rechts, in: Hof, Hagen/Schulte, Martin (Hrsg.), Wirkungsforschung zum Recht III, Baden-Baden, 2001, S. 33 - 43.

21 Vgl. Weber, Max, Wirtschaft und Gesellschaft, 5. Aufl. Tübingen 1990 (1. Aufl. 1921), S. 13.

22 Zur Kritik des Zweck-Mittel-Schemas vor allem: Homann, Karl, Die Interdependenz von Zielen und Mitteln, Tübingen 1980.

23 Von Hayek, Friedrich August, Die Ergebnisse menschlichen Handelns aber nicht menschlichen Entwurfs, in: ders. Freiburger Studien (1969) S. 97 - 107. 
für den zweckrationalen Einsatz von Instrumenten zur Steuerung sozialer Interaktionen darstellen. (3) Die Wechselwirkungen zwischen Mitteln und Zwecken werden systematisch ausgeblendet. Damit geraten Zirkularitäten zwischen Mitteln und Zwecken nicht in den Blick.

Das Fazit der hier angestellten Überlegungen ist ernüchternd. Für diejenigen, die versuchen vorherzusagen, wie Gerichte rechtliche Regelungen mit weiten Ermessensspielräumen auslegen werden, sinkt der Grad der Vorhersehbarkeit mit den Schwächen der verwendeten Interpretationsmethoden. Von da aus erscheint es rational, wenn die Regelungsadressaten nach solchen rechtlichen Gestaltungsmöglichkeiten suchen, bei denen diese Unsicherheit jedenfalls im Vergleich zu anderen Lösungen relativ verringert werden kann. Es kann dann durchaus sein, dass solche Entscheidungen von auBen betrachtet als irrational erscheinen. Aus Sicht der Entscheider handelt es sich um eine Strategie, den Grad der Unsicherheit in Bezug auf die Entscheidungsfolgen zu verringern.

\subsection{Weiterentwicklung der rechtswissenschaftlichen Interpretationsmethode}

Wenn die teleologische Interpretationsmethode mit dem Zweck-Mittel-Paradigma arbeitet und dabei positive Wirkungsanalysen einsetzen muß (müßte) und die Methodenkritik sich auf beide Aspekte dieses Ansatzes bezieht, liegt es nahe, zum einen das Zweck-Mittel-Paradigma aufzugeben und zum anderen die positive Wirkungsanalyse methodisch neu zu fundieren. Hier soll zuerst auf den möglichen Einsatz der Instrumente der Neuen Institutionenökonomik für vergleichende Wirkungsanalysen eingegangen werden; sodann soll überlegt werden, ob und wie das Zweck-Mittel-Paradigma durch eine geeignetere Vorgehensweise ersetzt werden kann. ${ }^{24}$

Werden Wirkungsanalysen im Rahmen der Interpretation rechtlicher Regelungen mit Hilfe des ökonomischen Ansatzes durchgeführt, heißt dies, (1) daß von der Annahme eigennutzorientierten Rationalverhaltens der Regelungsadressaten ausgegangen wird, (2) daß als handelnde Akteure auf die einzelnen Adressaten abgestellt wird, nicht auf Kollektive, und (3) daß die Wirkung der Wahlentscheidung zwischen verschiedenen Interpretationsvarianten auf Veränderungen der sozialen Interaktion der Regelungsadressaten beruht. Werden auf der Grundlage dieses methodischen Ansatzes Hypothesen über die Wirkungen der Interpretationsvarianten entwickelt, erfolgen diese als sogenannte Wenn-Dann-Aussagen. Wenn Interpretationsvariante A gewählt wird, folgt Wirkung X, wenn Interpretationsvariante B gewählt wird, folgt Wirkung Y. Aus diesem Nebeneinander zwischen zwei Wirkungsanalysen wird dann eine vergleichende Wirkungsanalyse, wenn die Hypothesen so umformuliert werden, daß nunmehr auf die Wirkung in Bezug auf eine bestimmte Zielsetzung abgestellt wird. Dann lassen sich beide Hypothesen dergestalt verknüpfen, daß nunmehr eine Hypothese wie folgt gefaßt werden kann: Wenn Interpretationsvariante $X$ - und nicht Interpretationsvariante Y - gewählt wird, verbessert/verschlechtert sich der Grad der Zielerrei-

$24 \mathrm{Zu}$ den folgenden Ausführungen ausführlich: Kirchner, § 4 Die ökonomische Theorie, a.a.O. (Fn. 14), S. $33-44$. 

schutz

chung in Bezug auf Ziel Z. Eine solche Hypothese beruht auf einer Analyse, die breiter angelegt war, also nicht nur auf die Frage der Zielerreichung des Zieles Z gerichtet war. Das ist deshalb wichtig, da in der Wirkungsanalyse auch andere Wirkungen der untersuchten Interpretationsvarianten Gegenstand der Analyse gewesen sind. Im Zweck-Mittel-Paradigma ist von >Nebenbedingungen $<$ die Rede. Verläßt man dieses Paradigma, geht es schlicht um unterschiedliche Wirkungen, die teils intendiert, teils nicht intendiert sind. Nun liegt aber eines der grundlegenden Probleme beim Versuch, Verhalten über Recht zu steuern, in den nicht intendierten Nebenwirkungen. Es muß sich aber keineswegs um nicht sintendierte < Nebenwirkungen handeln; es kann sich auch um solche Nebenwirkungen handeln, die zwar bekannt sind, die aber aus dem Entscheidungskalkül des Rechtsetzers ausgeblendet waren. Dies ist etwa bei $>\mathrm{Ne}-$ benfolgen sektorspezifischer Regulierung der Fall, die sich aus der Tatsache ergeben, daß einmal aufgebaute Bürokratieapparate nur schwer wieder abzuschaffen oder zu redimensionieren sind.

Gilt es, eine Wirkungsanalyse auf der Grundlage der ökonomischen Theorie, wie sie im Vorabschnitt eingeführt worden ist, in den rechtswissenschaftlichen Interpretationsansatz zu integrieren, sind zwei Probleme zu lösen: (1) Die Common-Sense-Analyse konventioneller Prägung ist durch eine ökonomische zu ersetzen. (2) An die Stelle des Zweck-Mittel-Paradigmas muß das Paradigma einer umfassenden vergleichenden Folgenabschätzung treten.

Wird die konventionelle Common-Sense-Analyse durch eine ökonomische ersetzt, bedeutet dies - wie betont - nicht einen einfachen Austausch eines Ansatzes durch einen anderen. Die rechtswissenschaftliche Interpretation als solche ist gestuft durchzuführen. Zuerst sind die juristisch-dogmatisch möglichen Interpretationsvarianten herauszufiltern. Erst dann können diese einer vergleichenden ökonomischen Wirkungsanalyse unterzogen werden.

Schwieriger gestaltet sich der Verzicht auf das Zweck-Mittel-Paradigma. Dessen methodische Mängel sind so gravierend, daß zwei radikale Änderungen geboten erscheinen. Die erste betrifft die Festlegung des Normziels, die sich als Einfallstor für nicht kontrollierbare Freiheiten des Interpreten erwiesen hat. An die Stelle der Festlegung des Interpreten auf eine eindeutige Zielsetzung der Norm hat eine Diskussion der Zielproblematik zu treten. Diese kann aber erst sinnvoll erfolgen, wenn die gegebenenfalls unterschiedliche Wirkungsweise verschiedener Interpretationsvarianten geklärt worden ist. Das hat zur Konsequenz, daß die Fragerichtung geändert wird. Es ist nicht vom Zweck auf die Mittel zu schließen. Vielmehr ist die Zieldiskussion im Lichte der Ergebnisse der vergleichenden Wirkungsanalyse zu führen. Werden in dieser Wirkungsanalyse, wie im Vorabschnitt betont, auch die nicht intendierten oder nicht in den Blick genommenen Wirkungen erfaßt, läßt sich die Zieldiskussion erheblich differenzierter führen. Dann können Ziele stärker ausdifferenziert werden, Nebenbedingungen und Kosten der Zielerreichung erörtert werden. Nicht intendierte Nebenwirkungen stellen sich oftmals als Sonderkosten der Zielerreichung ein. Wie normativ mit dem Problem umzugehen ist, das darin begründet liegt, daß Wechselwirkungen zwischen Zweck und Mitteln nicht zu verhindern sind, kann erst geklärt werden, wenn feststeht, auf welchen ökonomischen Ansatz zuzugreifen ist. 
Denn es macht einen Unterschied, ob in einem wohlfahrtsökonomisch ausgerichteten ökonomischen Ansatz die Zweck-Mittel-Diskussion mit Blick auf das Ziel effizienter Ressourcenallokation geführt wird oder ob in einem institutionenökonomischen Ansatz die normative Richtschnur der hypothetische Konsens ist.

Die Methodik der Interpretation rechtlicher Regelungen auf dem genannten ökonomischen Fundament stellt auf die erwarteten Folgen (Konsequenzen) unterschiedlicher Interpretationsvarianten $a b$, zwischen denen der Interpret wählt. Deshalb kann dieser Ansatz als >konsequentialistische Interpretationsmethode < bezeichnet werden. Weiß der Regelungsadressat, daß eine solche Interpretationsmethode zur Anwendung gelangt, wird seine Fähigkeit, Prognosen gerichtlicher Entscheidungen zu machen, deutlich verbessert. Die Ratio der Entscheidung tritt klarer zu Tage, als dies bei der herkömmlichen teleologischen Interpretationsmethode der Fall ist. Mit der Verringerung der Unsicherheit der Regelungsadressaten steigt zugleich die Rationalität ihrer Entscheidungen. Sie werden weiterhin mit dem Problem unvollständiger Information konfrontiert sein. Und sie werden weiterhin beschränkt rational entscheiden. Vorhandene Rationalitätsanomalien lassen sich allein durch die Wahl einer konsequentialistischen Interpretationsmethode nicht aus der Welt schaffen. Eine solche Wahl verbessert aber die Rationalität der gerichtlichen Entscheidungspraxis, wenn diese sich mit Problemen unbestimmter Rechtsbegriffe und mit Ermessensspielräumen in rechtlichen Regelungen zu befassen hat. 